Ann. Zootech., I964, 13 (I), 93-102

\title{
ÉTUDE DE LA TENDRETÉ DE LA VIANDE
}

\author{
I. - Comparaison des estimations de la TENDRETt́ \\ OBTENUES AVEC IE 《 WARNER BRATLZER APPARATUS 》 \\ ET AVEC UN JURY DE DÉGUSTATEURS
}

\section{Y. RENOU}

avec la collaboration technique de Colette GiRard

Laboratoire de Recherches sur la Viande, Centre national de Recherches zootechniques, Jouy-en-Josas (Seine-et-Oise)

\section{SOMMAIRE}

Cette étude rapporte les résultats d'une comparaison entre l'appréciation de la tendreté par un jury de dégustateurs $(\mathrm{T})$ et sa mesure $(\mathrm{W})$ à l'aide de l'appareil "WARNER-BRATZLER ».

L'étude de deux groupes de 108 et I35 échantillons de muscles a conduit à des coefficients de corrélation entre les deux mesures de $-0,670$ et $-0,768$ respectivement, correspondant aux équations de régression :

$$
\begin{aligned}
& \mathrm{W}_{1}=-\mathrm{I}, 26 \mathrm{~T}+\mathrm{I}_{5,28} \\
& \mathrm{~W}_{2}=-\mathrm{r}, 25 \mathrm{~T}+\mathrm{I}_{4,59} .
\end{aligned}
$$

Le caractère linéaire de la relation est discuté et l'hypothèse d'une liaison logarithmique de la forme $\mathrm{T}_{2}-\mathrm{T}_{1}=\mathrm{K} \cdot \log \frac{\mathrm{W}_{2}}{\mathrm{~W}_{1}}$ est envisagée.

Bien que hautement significative, la liaison entre les deux modes de mesures est loin d'être parfaite.

Ces résultats semblent justifier la poursuite des recherches d'une autre méthode mécanique de mesure de la tendreté. En attendant, il convient d'utiliser l'appareil avec une grande prudence et dans des conditions bien définies et bien précisées.

Il est permis, sans courir de grands risques d'erreurs, de donner à la tendreté la première place parmi les diverses caractéristiques organoleptiques des viandes.

La tendreté conditionne, en effet, l'appréciation des autres qualités telles que la saveur ou la jutosité ; en quelque sorte, " elle leur sert de support " (Dumonr, I952).

Ce fait n'est, en général, pas discuté et les résultats d'un sondage restreint de la population de la région parisienne l'ont récemment confirmé (RENOU, Ig63). 
Les revues de mise au point relatives à ce problème, tant en France (Dumont, I952 ; RENOU, I962) qu'à 1'étranger (HARRISON et al., I959; GRAVERT, I963), soulignent la multitude des facteurs, zootechniques et technologiques, qui peuvent influencer le degré de tendreté de la musculature d'un animal. Parmi ces facteurs, les principaux semblent être l'âge de l'animal, son alimentation, le mode d'élevage auquel il a été soumis et surtout les modes de conservation et de cuisson adoptés.

Il va de soi, et les revues précitées le soulignent, que le nombre de ces facteurs et leur interaction possible rendent difficile l'analyse de l'influence de chacun.

Cette difficulté est encore considérablement accrue par le fait que la tendreté - qualité subjectivement discriminable - doit, pour être étudiée, être mesurée. De fait, le choix des méthodes de mesure ou d'appréciation reste, dans ce domaine, le problème majeur.

Les diverses méthodes de mesure, tant subjectives qu'objectives, ont retenu l'attention de nombreux auteurs (Dumont, I952; Renou, I962; PEARSON I963). Le nombre des méthodes objectives proposées est très élevé. Leurs promoteurs, comme leurs utilisateurs, sont d'accord sur la nécessité de confronter ces méthodes objectives avec l'appréciation subjective des caractères mesurés, la première qualité d'une méthode objective devant être, en effet, de mesurer effectivement le caractère considéré, tel que peut l'apprécier le consommateur.

Cette étude expose les résultats obtenus au cours d'une utilisation comparative d'une méthode objective de mesure de la dureté de la viande ("Warner-Bratzler Shear-Force Apparatus ") et d'une méthode strbjective (jury de dégustation).

\section{MATÉRIEL ETT TECHNIQUES}

L'étude a porté sur un ensemble de 243 muscles prélevés sur les carcasses de 27 bovins abattus au C. N. R. Z. de septembre 1962 à mars 1963 et répartis en 2 séries de 12 et 15 animaux respectivement. Ces 2 séries ne représentent pas 2 lots expérimentaux différents pour la présente étude. Elles correspondent seulement à une division chronologique ayant pour but de permettre l'appréciation de la fidélité du travail des dégustateurs dans le temps.

\section{A) Préparation des échantillons}

Après un ressuyage de 24 heures, les demi-carcasses étaient découpées et l'ensemble épaulecollier de la demi-carcasse gauche prélevé, puis stocké en chambre froide à $+4^{\circ} \mathrm{C}$, jusqu'au jour du prélèvement des muscles. Ce prélèvement avait lieu le septième jour après l'abattage, c'est-ì-dire la veille du jour des tests de dégustation.

Neuf muscles de l'ensemble épaule-collier ont été retenus pour cette étude :

$$
\begin{aligned}
\text { dans l'épaule : } & \text { - le Grand rond (Teres major); } \\
& \text { - le Sus-épineux (Supraspinatus); } \\
& \text { - le Sous-épineux (Infraspinatus); } \\
& \text { - le Gros anconé (Caput longum tricipitis brachii); } \\
\text { dans le collier : } & \text { - le Rhomboïde (Rhomboïdeus); } \\
& \text { - le Splénius (Splenius); } \\
& \text { - le Grand complexus (Semi spinalis capitis); } \\
& \text { - le Dentelé du cou (Levator scapulae). }
\end{aligned}
$$

L'intérêt de ce groupe de muscles est de présenter une gamme de duretés étendue. On y trouve, en effet, des muscles normalement destinés à être grillés (Grand rond) et des muscles destinés à être braisés ou bouillis (Grand complexus, Rhomboïde). 


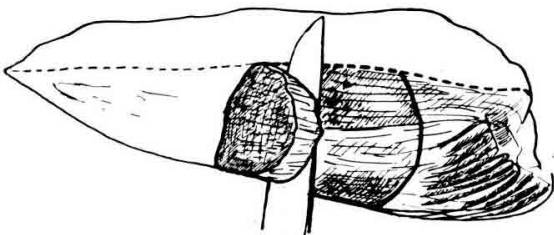

m. supraspinatus

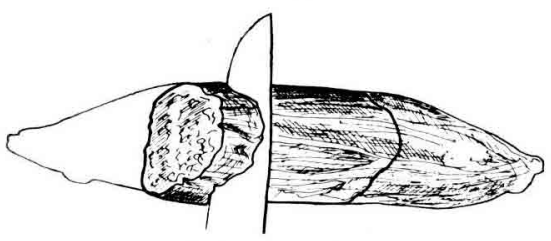

m. teres major

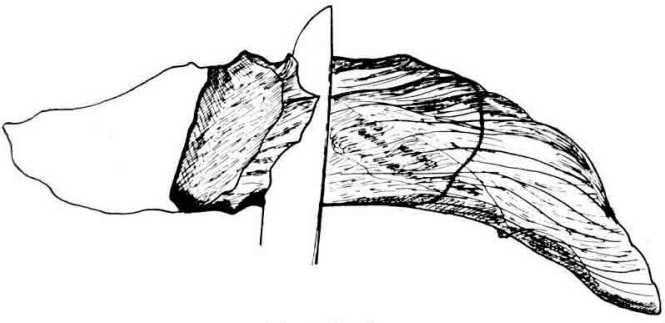

m. splenius

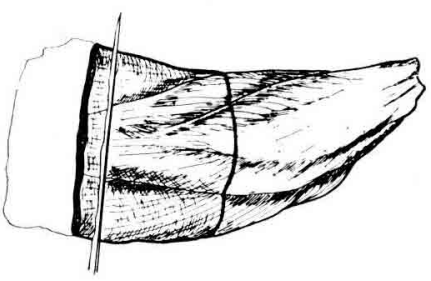

m. semi spinalis capitis.

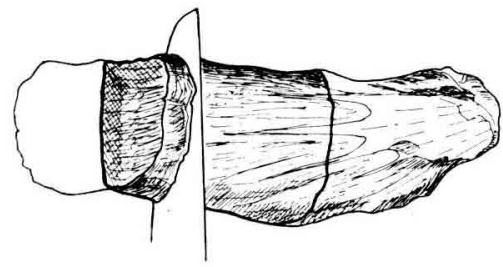

m. infraspinatus

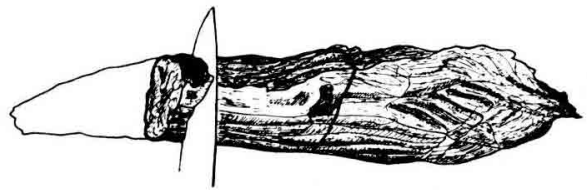

m. rhomboïdeus

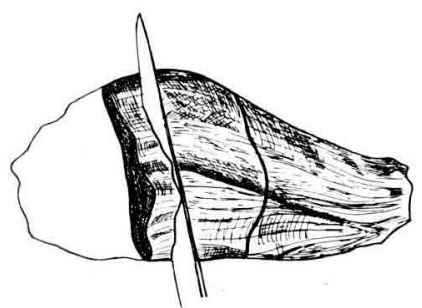

m. triceps brachii. (cap. laterale)

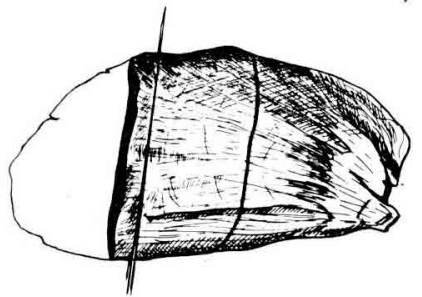

m. triceps brachii (cap. longum)

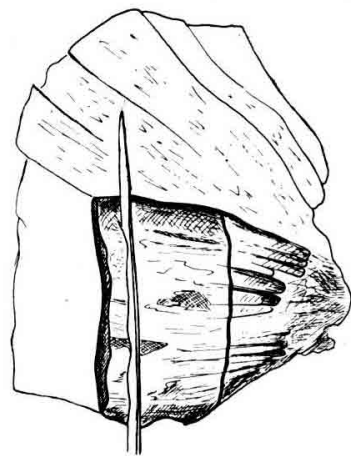

m. 1 evator scapulae ANNEXE. - Schémas des neuf muscles étudiés. Sur chacun des muscles sont représentés
la portion de muscle considérée dans l'étude (contour rentorcé) et le sens de coupe des tranches
(indiqué par l'inclinaison de la lame du cowteuu) 
Ces 9 muscles, isolés la veille de la dégustation vers I $7 \mathrm{~h}$ étaient ensuite parés, le matin même, afin qu'y soient prélevées les tranches destinées à la cuisson.

Ces tranches, épaisses d'environ $12 \mathrm{~mm}$, étaient, pour chacun des 9 muscles, coupées d'une manière constante, en particulier en ce qui concerne l'axe de coupe par rapport aux fibres musculaires (cf.. schémas en annexe).

Les tranches étaient cuites sur un gril à gaz pendant $2 \mathrm{mn}$ pour chaque face. Les mêmes tranches servaient à la détermination de la tendreté par les deux méthodes, objective et subjective.

\section{B) Appréciation subjective}

Les tranches ainsi cuites étaient découpées en " bouchées " carrées d'environ I5 $\mathrm{mm}$ de côté et chaque dégustateur recevait pour chaque échantillon 2 de ces bouchées de viande. Les 9 muscles étaient répartis en 3 séries de 3 échantillons codés A, B, C, soumis à un test combiné de classement et de cotation par rapport à l'échelle ci-dessous :

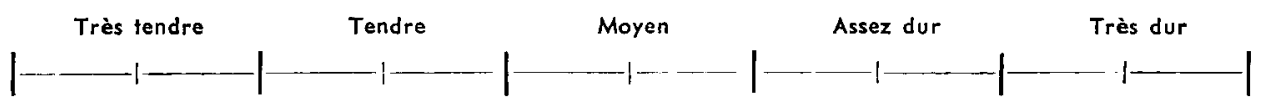

Pendant la dégustation, les dégustateurs étaient placés dans des isoloirs. Le jury utilisé était composé de 9 membres entraînés. Une étude préliminaire ayant porté sur 99 échantillons de viande provenant de i I animaux, étudiés pendant les mois de janvier et février I962, avait permis de sélectionner ces 9 dégustateurs à partir d'un ensemble de is personnes. La constance de la position de chaque dégustateur par rapport à la moyenne du jury a été contrôlée (ce travail de sélection fera l'objet d'une étude particulière actuellement en préparation). Par ailleurs, l'introduction de doubles dans ces séries préliminaires a permis d'évaluer la fidélité de ces dégustateurs. Ces deux critères, ajoutés à l'exigence d'une bonne assiduité aux séances de dégustation, ont permis de retenir les 9 jurés dont les réponses sont utilisées dans cette étude.

Ces réponses ont été ensuite transformées linéairement en notes de I à Io, dans l'ordre des tendretés croissantes. A partir des 9 notes ainsi attribuées à chaque échantillon a été calculée une note moyenne de tendreté (T).

\section{C) Mesure de l" "indice mécanique de dureté" à l'aide de l'appareil de Warner et Bratzler}

En même temps que les " bouchées " destinées aux dégustations et dans les mêmes tranches cuites, étaient prélevées, parallèlement au sens des fibres, des "carottes " de $12,7 \mathrm{~mm}$ de diamètre (I/2 inch) destinées à la mesure de l'indice mécanique. Dans chaque échantillon a ainsi été prélevé un nombre de carottes égal à $\mathrm{i} 2 \pm 2$. La moyenne de toutes les mesures exprimées en livres anglaises ( $\left.{ }^{1}\right)$ était utilisée comme indice de dureté de chaque muscle (W).

\section{RÉSUL'TATS}

Les 243 échantillons ont obtenu des notes de tendreté allant de 2,5 à 9,5 et présenté des indices mécaniques variant de 2,5 à 23,0 lbs.

En ce qui concerne l'appréciation subjective, les dégustateurs ont utilisé convenablement la totalité de l'échelle proposée, avec une bonne sensibilité. De même, la fidélité de ce jury a été bonne, puisque pour I3 doubles, l'écart entre 2 notes accor-

(1) L'appareil de Warner et Bratzler étant de construction américaine, nous avons utilisé pour le prélivement des "carottes " les accessoires américains. Pour cette même raison, les résultats ont été conservés en mesures anglaises, tels que les donne la graduation de l'appareil. 
dées par un juré donné aux 2 échantillons d'un même morceau est toujours resté inférieur à I unité et que cet écart pour la note moyenne T n'a jamais dépassé 0,5 .

L'analyse de variance des mesures de ces échantillons doubles est résumée, pour les 2 méthodes de mesure, dans les tableaux I et 2.

TABLEAU I

Notes de dégustation

\begin{tabular}{l|r|c|c|c}
\hline \hline $\begin{array}{c}\text { Origine de la } \\
\text { variation }\end{array}$ & $\begin{array}{c}\text { Somme } \\
\text { des carrés }\end{array}$ & $\begin{array}{c}\text { Degrés de } \\
\text { liberté }\end{array}$ & Variance & F \\
\hline Traitement ... & 24,7589 & 12 & 2,0632 & $23,1\left({ }^{1}\right)$ \\
Erreur ...... & 1,1615 & 13 & 0,0893 & \\
Total ....... & 25,9204 & 25 & & \\
\hline
\end{tabular}

TABLEAU 2

Indices mécaniques

\begin{tabular}{|c|c|c|c|c|}
\hline $\begin{array}{c}\text { Origine de la } \\
\text { variation }\end{array}$ & $\begin{array}{l}\text { Somme } \\
\text { des carrés }\end{array}$ & $\begin{array}{c}\text { Degrés de } \\
\text { liberté }\end{array}$ & Variance & $\mathrm{F}$ \\
\hline $\begin{array}{l}\text { Traitement ... } \\
\text { Erreur ...... } \\
\text { Total ....... }\end{array}$ & $\begin{array}{r}122,9553 \\
23,0660 \\
146,0213\end{array}$ & $\begin{array}{l}12 \\
13 \\
25\end{array}$ & $\begin{array}{r}10,2462 \\
1,7743\end{array}$ & $5,8\left({ }^{1}\right)$ \\
\hline
\end{tabular}

(1) Valeur tabulaire de $\mathrm{F}$ pour $\mathrm{P}=0,01: 3,96$.

On peut donc constater une bonne répétabilité ainsi estimée des deux méthodes de mesure, avec une légère supériorité de la dégustation.

Cette supériorité se trouve confirmée par les estimations des coefficients de fidélité de la forme :

$$
\mathrm{R}=\frac{\mathrm{S}_{x}^{2}}{\mathrm{~S}_{x}^{2}+\mathrm{S}_{e}^{2}}
$$

et qui sont, respectivement, 0,95 pour la dégustation et 0,85 pour la mesure mécanique Corrélation entre les deux mesures.

Pour chacun des deux groupes d'animaux, comportant respectivement Io8 et I35 échantillons, une corrélation linéaire a pu être mise en évidence (test de linéarité, cf. VESSEREAU) (fig. I $a$ et $b$ ).

Les coefficients de corrélation calculés ont été respectivement :

$$
\begin{aligned}
& r_{1}=-0,670 \pm 0,05 \\
& r_{2}=-0,768 \pm 0,07 .
\end{aligned}
$$

Annales de Zootechnie. - I964. 
Ces valeurs sont hautement significatives et confirment les résultats généralement rencontrés dans la littérature (revues déjà citées).

Toutefois, il faut noter que 5 seulement des 9 coefficients de corrélation partiels calculés pour chacun des muscles étudiés, c'est-à-dire pour des notes présentant une variabilité plus faible, sont significatifs ou hautement significatifs.

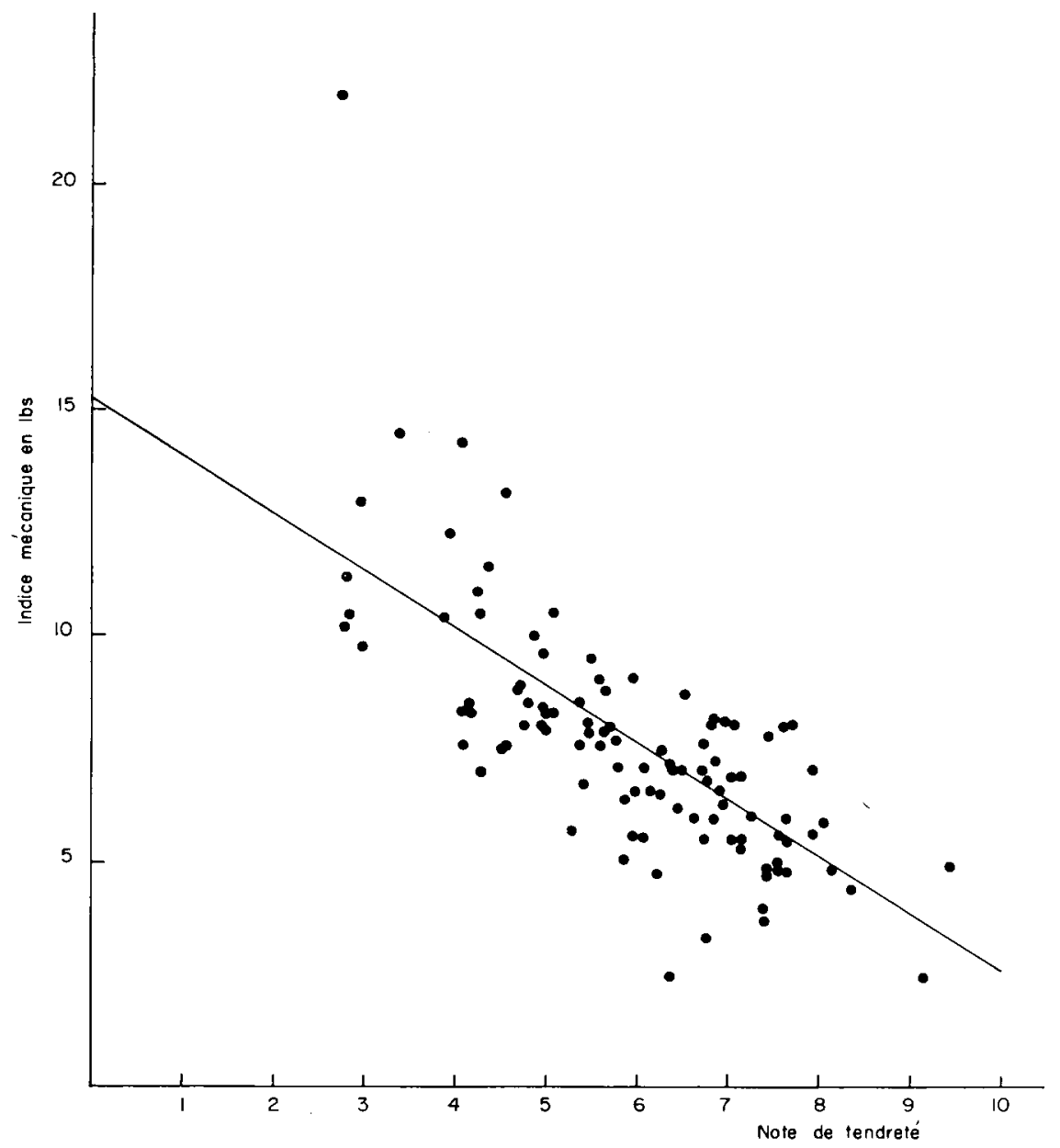

Fig. I a. - Relalion enlre les indices de durelé el les notes de tendreté des échunlillons de muscles du premier groupe d'animanx

Lorsqu'on considère globalement les io8 et les I35 échantillons des deux groupes d'animaux étudiés, on obtient les deux équations de régression suivantes:

$$
\begin{aligned}
& W=-I_{2} 6^{\prime} \mathrm{I}^{\prime}+\mathrm{I}_{5,28} \\
& W=-\mathrm{I}, 25 \mathrm{I}^{\prime}+\mathrm{I}_{4,59}
\end{aligned}
$$

Cet accord entre les deux séries de résultats nous paraît pouvoir être considéré comme une confirmation de la qualité du travail des dégustateurs, puisque malgré 
le décalage dans le temps, la relation avec la mesure mécanique est restée sensiblement la même.

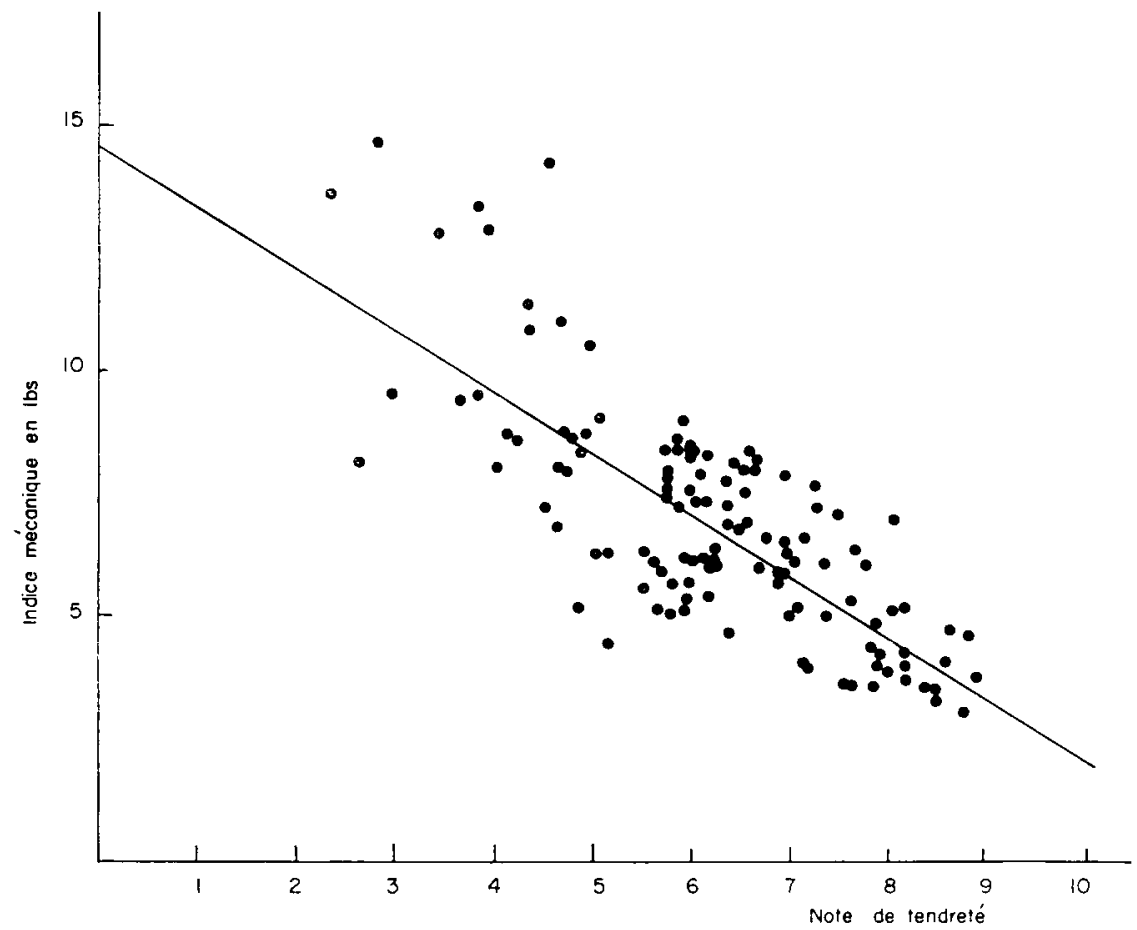

FIs. г. $b$ - Relation entre les indices de dureté et les notes de tentreté des échantillons de muscles du deuxiène groupe d'animaux

\section{DISCUSSION - CONCLUSION}

Ces résultats confirment donc qu'une corrélation négative hautement significative peut être mise en évidence entre les deux modes d'appréciation de la tendreté de la viande.

Le calcul montre que cette relation peut être considérée comme linéaire. Toute fois, on peut remarquer graphiquement que la linéarité est moins bonne dans la zone des indices élevés, c'est-à-dire pour les viandes dures.

On peut donc émettre 1'hypothèse qu'entre la dureté de la viande (objectivement mesurée par son indice mécanique) et la sensation de dureté ou de tendreté (subjectivement exprimée par le dégustateur), il existerait plutôt une relation du type semi-logarithmique, comme sont généralement les relations psycho-physiques entre les sensations et les stimuli qui les provoquent, c'est-à-dire de la forme :

$$
\mathrm{T}_{2}-\mathrm{T}_{1}=\mathrm{K} \cdot \log \frac{\mathrm{W}_{2}}{\mathrm{~W}_{1}} \text {. }
$$

En fait, si on transforme les indices mécaniques en leur logarithme et qu'on étudie la relation entre la note de tendreté et le logarithme de l'indice mécanique, 
on constate une meilleure linéarité de la relation que dans le calcul frécédent. Cette relation, calculée pour la première série de Io8 échantillons serait:

$$
\text { 'I' }=-\mathrm{II}, 5 \log . \mathrm{W}+\mathrm{I} 6 .
$$

Une telle relation, dans le domaine de la dureté de la viande, rejoindrait l'hypothèse de WEBER et la loi de WEBER-FECHNER bien connues en psychophysiologie sensorielle.

'Toutefois, d'un point de vue pratique, il faut remarquer (fig. 2) que les deux relations ainsi défnies (relation linéaire et relation semi-logarithmique) conduisent à des courbes sensiblement confondues pour la zone de tendreté 4-9 (correspondant à des indices mécaniques de 10,5 à 4), c'est-à-dire four la zone de l'échelle où se trouvent la majorité des muscles de bovins. On peut donc considérer comme suffi-

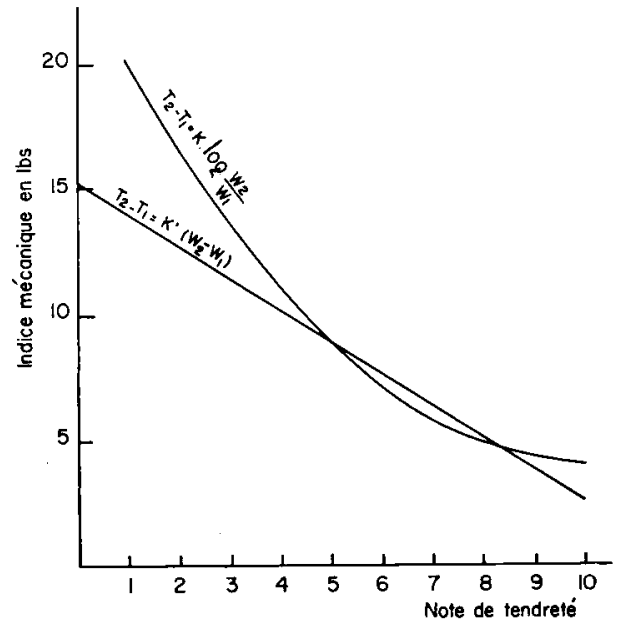

FIG. 2. - Comparaison des relations linéaire et semi-logarithrique entre indice mécanique de dureté et nole de tenilreté

sante la relation linéaire simple et admettre l'accord entre les deux méthodes de mesure. Il ne faut toutefois pas perdre de vue que si la corrélation statistique entre les 2 méthodes est élevée, il n'en reste pas moins que leur accord n'est pas parfait, surtout pour la zone des duretés élevées. Ceci ne saurait surprendre, puisqu'on sait que la résistance mécanique ne représente qu'un des aspects de la dureté.

I1 faut, par ailleurs, noter, d'une part, la variabilité de dureté dans un même muscle, représentée par la dispersion des mesures mécaniques pour un même morceau et, d'autre part, la variabilité de l'appréciation, dans l'absolu, de cette dureté pour le consommateur.

Cette constatation oblige à souligner à nouveau l'extrême importance du choix et de la préparation des échantillons étudiés.

En ce qui concerne les méthodes de mesure, l'appareil de WARNER et BRATZI.ER a été, et est encore, largement utilisé. C'est, avant tout, cette raison qui nous a poussés à l'utiliser et à l'étudier.

L'existence d'une corrélation significative entre les résultats qu'il donne et 
ceux obtenus par la dégustation permet de considérer que cet appareil donne une estimation indicative de la dureté. Ce résultat positif justifie surtout la poursuite des recherches d'une méthode de mesture mécanique.

Par ailleurs, malgré la différence de sensibilité au facteur " dureté " possible entre nations différentes, cette corrélation est en accord avec les résultats donnés dans la littérature étrangère (PEARson, I963). Ceci permet de penser que l'appareil mesure bien un facteur constant lié à la sensation de dureté, même si on ne peut prétendre par là mesurer la tendreté, au sens que lui attribue le consommateur.

Malgré tout, cet appareil continue d'être l'objet de critiques multiples (MIYADA et TAPpel, I956; Michaud, I963). Ces auteurs reprochent à la méthode son mode d'échantillonnage qui fait que l'on mesure la force mécanique dans une seule direction. On peut également lui reprocher, en liaison avec ce mode d'échantillonnage et surtout du fait de la nature du travail enregistré, de ne considérer qu'un aspect du processus de mastication. Ces critiques paraissent, dans l'ensemble, être justifiées et la variabilité enregistrée ici le confirme.

On peut donc continuer, en attendant de disposer d'une méthode supérieure au Warner-Bratzler - et, si possible, aussi simple et aussi rapide que lui — à l'utiliser pour évaluer la dureté mécanique de la viande. Mais il ne faut surtout pas oublier que cette méthode nécessite un nombre élevé de mesures (Michaud, rg63) et que l'indication fournie ne semble pouvoir exprimer la tendreté que pour des valeurs moyennes de cette caractéristique.

Reģu pour publication en décembre $\mathbf{1 9 6 3 .}$

\section{SUMMARY}

BEEF TENDERNESS. I. OBJECTIVE AND SUBJECTIVE MEASUREMENT. CORRELATION BETWEEN SHEAR VALUES AND TASTE-PANEL SCORES

Beef tenderness evaluation (T) by a trained taste panel and its measurement (W) by the WARNER-BRATZLER shear apparatus were compared in a study of 2 groups of 108 and I 35 muscle samples. The calculated correlation coefficients for these 2 groups of measurements were $-0,670$ and $-0,768$ respectively, with the following corresponding linear regressions :

$$
\begin{aligned}
& \mathrm{W}_{1}=-\mathrm{I}, 26 \mathrm{~T}+\mathrm{I} 5,28 \\
& \mathrm{~W}_{2}=-\mathrm{I}, 25 \mathrm{~T}+\mathrm{I}, 59
\end{aligned}
$$

The linearity of the relation is discussed and so is a possible semi-logarithmic relation such as $\mathrm{T}_{2}-\mathrm{T}_{1}=\mathrm{K} \cdot \log \frac{\mathrm{W}_{2}}{\mathrm{~W}_{1}}$.

Though highly significant, the relation between the two methods is not perfect and it is concluded that carrying on research to find a better objective measurement is useful.

Before such an apparatus is found, the "Warner-Bratzler " may be used to obtain indications of meat toughness; in any case this apparatus must be used with extreme care and the sampling methods must be very well described.

\section{RÉFÉRENCES BIBLIOGRAPHIQUES}

Dumont B., I952. La tendreté de la viande. Ann. Zootech., 3, 71-95.

Gravert H. O., 1963. Untersuchungen über die Erblichkeit von Fleischeisenschaften beim Rind. II. Mitteil. Eiweissgehalt, $\mathrm{pH}-$ Wert, Farbe, Muskelfaserstäke, Zartheit. Z. Tiersuch. Ziichtungsbiol., 78, I $39^{-1} 78$. 
Harrisson D. L., Visser R. and Sister Rosetta Schirmer. 1959. A resume of the litterature related to factors affecting the tenderness of certain beef muscles. Ioth Report. Kansas Agricullural Exp. Station.

Michald C., ig63. Méthodes de mesure de la tendreté de la viande de boucherie. Thèse Doct. Vét., Alfort.

Miyada D. S. et ThPPel A. L., 1956. Meat Tenderization. I. Two mechanical devices for measuring texture. Food Technol., 10, I $42-45$.

Pearson A. M., ig63. Objective and subjective measurements for meat tenderness. Proc. Campbell Soup Co. Meat Tenderness Symposium. Camden.

Renot I., I962. Les qualités organoleptiques des viandes. Ann. Nul. Alim., 16, (3) I-58.

Renou Y., I963. Note sur les résultats des épreuves de consommation organisées à l'occasion du Concours Général Agricole. In La Cité de la Viande. B. T. I. Ed. Paris.

Steel R. G. D. et Torrie J. I1., ig6o. Principles and procedures of statistics. Mc Graw, Hill Book Co ed., New York.

Vessereau A., I948. Méthodes statistiques en biologie et en agronomie. J. B. Baillère et Fils Éd., Paris. 